\section{A Rapid-Cycle Quality Improvement Initiative to Increase Compliance with Infection Control Precautions in a Pediatric Ward}

To the Editor-We read with interest the study by Fries et al ${ }^{1}$ on the best way to sample hand hygiene compliance using human observers. They explored how hand hygiene observer scheduling influences the number of events and unique individuals observed and found that short observation periods of 15 minutes each provided the best estimator of compliance for the shift. They also found that the first hour of each day shift, which corresponded with morning rounds, provided the best overall estimator of the entire shift. These results support the methods used in our quality improvement (QI) project, conducted on a much smaller scale, where observations were carried out for short periods and concentrated during the day shift, particularly morning rounds. We sought to measure rates of compliance with infection control precautions in a 36-bed pediatric ward in a 496-bed urban academic medical center. Although intervention studies to increase hand hygiene compliance have not provided conclusive results, ${ }^{2,3}$ QI methods, including rapid improvement approaches, ${ }^{4}$ have been shown to result in sustained improvement. ${ }^{5}$ Therefore, our secondary aim was to determine whether a focused educational program with feedback would improve these rates.

Over an 8-week period, from mid-December 2011 to early February 2012, monitors directly observed clinical and ancillary staff entering and exiting patient rooms. Monitors observed over 30-minute intervals during the weekday shift. Patient room, type of infection control precaution, whether the infection control precautions were carried out in the correct sequence, healthcare worker (HCW) job category (registered nurse $[\mathrm{RN}]$, resident, attending), training level of med-

TABLE 1. Preintervention and Postintervention Overall Total Compliance with Standard and Contact Precautions

\begin{tabular}{lcc}
\hline & $\begin{array}{c}\text { Preintervention } \\
(n=98)^{\mathbf{a}}\end{array}$ & $\begin{array}{c}\text { Postintervention } \\
(n=54)^{\mathbf{b}}\end{array}$ \\
\hline MDs/MDs-in-training & 55 & 51 \\
Medical students & 100 & 100 \\
Residents/fellows/clinicians & 62 & 35 \\
Attendings & 26 & 46 \\
RNs & 33 & 29 \\
All HCWs & 47 & 44
\end{tabular}

NoTE. Data are percentage of total compliance. HCW, healthcare worker; $\mathrm{MD}$, medical doctor; $\mathrm{RN}$, registered nurse.

a 38 entering, 60 exiting.

b 27 entering, 27 exiting. ical doctor (MD) if known, and sex were recorded. The intervention consisted of a 1-week period of 1 focused, intensive, interdisciplinary 40-60-minute session for MDs led by the fellow and infection control nurse; this consisted of a 10-minute video case study followed by discussion, education on the standards and logistics of infection control, and testing and feedback. Two additional sessions were carried out by the fellow to reinforce logistic details and to answer questions. RNs were given repeat, short teaching sessions daily throughout the week by the nurse manager during their daily huddle. Postintervention observations were carried out the following week in March 2012.

Analysis was conducted using SAS, version 9.1 (SAS Institute). Comparisons were made using a $\chi^{2}$ test of independence where appropriate, with statistical significance set at $P<.05$.

Ninety-eight events were observed during the preintervention phase (38 entering, 60 exiting). Overall compliance with standard and contact precautions among HCWs was $47 \%$ (55\% among MDs, 33\% among RNs; see Table 1). Fifty-seven percent sanitized their hands upon entering or exiting patient rooms, with a higher percentage doing so on exiting (67\%) than on entering ( $42 \% ; P=.017$; see Table 2$)$.

A total of 54 events ( 27 entering, 27 exiting) were observed during the immediate postintervention period (Table 1). Overall compliance with standard and contact precautions was $51 \%$ for MDs and $29 \%$ for RNs. Overall compliance with hand hygiene was 50\% (59\% exiting, $41 \%$ entering; $P=$ .17; Table 2).

Adherence to infection control precautions, in particular hand hygiene, is vital in preventing the spread of infection and has significant implications for nosocomial infection rates and HCW safety. ${ }^{6}$ The results from our rapid-cycle QI initiative highlight the opportunity to improve HCW compliance with infection control precautions in the study unit. There was no significant difference in overall compliance before and after a brief but intensive focused intervention $(P=.77)$. There was also no improvement in overall hand hygiene rates $(P=.4)$. The trend toward higher hand hygiene compliance upon exiting has been demonstrated in previous studies and attributed to HCW desire for self-protection rather than for patient safety. ${ }^{7}$ Similarly, the lower rates of

TABle 2. Preintervention and Postintervention Hand Hygiene Compliance

\begin{tabular}{|c|c|c|c|c|c|c|}
\hline & \multicolumn{3}{|c|}{$\begin{array}{l}\text { Preintervention } \\
\quad(n=98)\end{array}$} & \multicolumn{3}{|c|}{$\begin{array}{l}\text { Postintervention } \\
\quad(n=54)\end{array}$} \\
\hline & $\begin{array}{l}\text { Entering } \\
(n=38)\end{array}$ & $\begin{array}{l}\text { Exiting } \\
(n=60)\end{array}$ & $P$ & $\begin{array}{l}\text { Entering } \\
(n=27)\end{array}$ & $\begin{array}{c}\text { Exiting } \\
(n=27)\end{array}$ & $P$ \\
\hline All HCWs & 42 & 67 & .017 & 41 & 59 & .17 \\
\hline Total & \multicolumn{2}{|c|}{57} & & \multicolumn{2}{|c|}{50} & .4 \\
\hline
\end{tabular}

NOTE. Data are percentage of hand hygiene compliance. 
compliance among nursing staff have also been described previously. ${ }^{8}$

The limitations of our study were the small sample size and monitoring that did not take into account what occurred inside the patient rooms. In addition, MDs and RNs received instruction from different personnel, so there may have been qualitative differences in the intervention received. Nevertheless, this project shows that despite the use of a rapidcycle intervention to improve quality of care, improvement will require a concerted and sustained effort that goes beyond education and feedback.

\section{ACKNOWLEDGMENTS}

We thank James Moses for his assistance in the design of this project. We also thank Karan Barry, Bob Burke, and Mohamed Dassouli for their participation in the quality improvement initiative sessions and Karan Barry for leading educational sessions with the registered nurses.

Potential conflicts of interest. All authors report no conflicts of interest relevant to this article. All authors submitted the ICMJE Form for Disclosure of Potential Conflicts of Interest, and the conflicts that the editors consider relevant to this article are disclosed here.

\section{Pui-Ying Iroh Tam, MD; ${ }^{1, a}$ Amy Bettano, MPH; ${ }^{2, b}$ Zoon Wangu, MD; ${ }^{1}$ Dale Ford, RN, MPH; ${ }^{3}$ Carol Sulis, $\mathrm{MD}^{4}$}

\begin{abstract}
Affiliations: 1. Division of Pediatric Infectious Diseases, Boston Medical Center, Boston, Massachusetts; 2. Department of Epidemiology, Boston University School of Public Health, Boston, Massachusetts; 3. Infection Control Department, Boston Medical Center, Boston, Massachusetts; 4. Division of Infectious Diseases, Boston Medical Center, Boston, Massachusetts; a. Present affiliation: University of Minnesota, Minneapolis, Minnesota; b. Present affiliation: Epidemiology Consult Division, United States Air Force School of Aerospace Medicine, Dayton, Ohio.

Address correspondence to Carol Sulis, MD, Epidemiology Unit, Dowling 3N, Boston Medical Center, 818 Harrison Avenue, Boston, MA 02118 (carol.sulis@bmc.org).

Presented in part: ID Week; San Diego, California; October 2012. Infect Control Hosp Epidemiol 2013;34(2):213-214

(C) 2013 by The Society for Healthcare Epidemiology of America. All rights reserved. 0899-823X/2013/3402-0018\$15.00. DOI: $10.1086 / 669075$
\end{abstract}

\section{REFERENCES}

1. Fries J, Segre AM, Thomas G, Herman T, Ellingson K, Polgreen PM. Monitoring hand hygiene via human observers: how should we be sampling? Infect Control Hosp Epidemiol 2012;33(7):689695.

2. Gould DJ, Moralejo D, Drey N, Chudleigh JH. Interventions to improve hand hygiene compliance in patient care. Cochrane $\mathrm{Da}$ tabase Syst Rev 2010;(9):CD005186.

3. Pittet D. Improving compliance with hand hygiene in hospitals. Infect Control Hosp Epidemiol 2000;21(6):381-386.

4. Martin SC, Greenhouse PK, Kowinsky AM, McElheny RL, Petras CR, Sharbaugh DT. Rapid improvement event: an alternative approach to improving care delivery and the patient experience. J Nurs Care Qual 2009;24(1):17-24.

5. Linam WM, Margolis PA, Atherton H, Connelly BL. Qualityimprovement initiative sustains improvement in pediatric health care worker hand hygiene. Pediatrics 2011;128(3):e689-e698.
6. Pessoa-Silva CL, Hugonnet S, Pfister R, et al. Reduction of health care associated infection risk in neonates by successful hand hygiene promotion. Pediatrics 2007;120(2):e382-e390.

7. Erasmus V, Brouwer W, van Beeck EF, et al. A qualitative exploration of reasons for poor hand hygiene among hospital workers: lack of positive role models and of convincing evidence that hand hygiene prevents cross-infection. Infect Control Hosp Epidemiol 2009;30(5):415-419.

8. De Wandel D, Maes L, Labeau S, Vereecken C, Blot S. Behavioral determinants of hand hygiene compliance in intensive care units. Am J Crit Care 2010;19(3):230-239.

\section{Reply to Iroh Tam et al}

To the Editor-We thank Iroh Tam et al ${ }^{1}$ for responding to our work on hand hygiene sampling. ${ }^{2}$ Indeed, one of our goals was to encourage more data-driven approaches to inform hand hygiene programs. In our original article, we collected and used spatially and temporally dense "sensor-mote" data to study healthcare worker (HCW) movement and interaction. We used these data to determine where and when to observe hand hygiene behavior in order to best measure compliance (as well as to answer other healthcare-related questions; see Hornbeck et $\mathrm{al}^{3}$ ). Intuitively, periods aligning with the start of shifts or morning rounds seem like good candidates for observation - an intuition our sensor data confirmed is true for the University of Iowa Hospitals and Clinics (UIHC) medical intensive care unit (MICU).

One possible criticism of our previous work relates to the generalizability of our results - that is, how our results can be reliably applied to other healthcare facilities outside the UIHC MICU without sensor-mote data. Because we recognize that it is not feasible for every healthcare facility to replicate our data collection and analysis methods, we propose another simple, inexpensive, data-driven methodology for selecting candidate hours for hand hygiene observation. When validated against our original fine-grained sensor-mote data, we find that this new approach performs very well, resulting in a 1.5-3-fold improvement over just randomly choosing hours for observation (with uniform density).

Our new method relies on using HCW log-ins to electronic health records (EHRs) as a proxy indicator for HCW location and level of activity. Because accessing patient EHR information has become a standard component of patient care, many healthcare facilities now place computer terminals in patient care areas or even in individual patient rooms. Historical records of HCW log-ins are easily retrievable from EHR systems and typically contain the time, user identifiers, and computer terminal identifiers for every log-in event and thus, by extension, the approximate location of the event. In other work, we show how HCW contact networks inferred from such log-in data approximate the quality of networks obtained with more accurate but expensive sensor-mote deployments. ${ }^{4}$ This same idea-that log-in records capture 